\title{
Components of high-performance computing and communication in the behavioral sciences
}

\author{
JOHN E. MERTZ \\ Minnesota Supercomputer Center, Inc., Minneapolis, Minnesota
}

\begin{abstract}
By its very nature, the spectrum of computational problems approached in the behavioral sciences necessitates the use of almost all elements of high-performance computing and communication. The salient performance issues of various components - networking, disk and tape storage, memoryprocessor configurations, and processing elements - are discussed. Hardware, software, and application issues surrounding parallel computing performance are also discussed.
\end{abstract}

If a researcher is performing an analysis on a large amount of remotely located data, these data must first be transferred across the network to the user's local file system. Then, to perform the computations, the data must be transferred into the central memory and then again transferred to the processor. Throughout this process, the performance delivered to the user is only as high as the slowest link in the hardware and software chain. The network may be the performance bottleneck, or the memory on the computer may not be large enough to prevent storing portions of the executing program on disk (i.e., thrashing).

The above example illustrates a few of the important elements of high-performance computing. In the text that follows, these elements will be highlighted along with a description of their performance characteristics.

\section{Networking Performance}

The exponential growth of data traffic on the Internet continues to outpace its supporting hardware and software. This is due, in part, to the increased number of users and, in part, to the ability of individual users to transfer virtually any quantity of data without charge. Because there is a lack of "metering" on a per-byte basis, there is a fundamental difference in the economics of the Internet and other utility industries. This makes the prediction of future Internet-capacity changes tenuous.

The sophisticated systems in place to dynamically route and reroute data paths make it difficult to predict transfer rates at any one sitting, but users of this shared resource should not expect more than a few megabit/second datatransfer rates. It is worth noting that to help ensure pre-

The author's address is Minnesota Supercomputer Center, Inc., 1200 Washington Ave. South, Minneapolis, MN 55415 (e-mail: jem@ msc.edu). dictable performance, many organizations elect to lease a dedicated communications channel.

On a more local level, users can make use of networking technology capable of $128 \mathrm{Kbits} / \mathrm{sec}$ (ISDN) to 1,000 Mbits/sec (fibre channel; Mayer, 1996). As of March 1996 , individual users could purchase ISDN modems for under $\$ 600$ and lease ISDN lines for under $\$ 200$ per month (Tadjer, 1996). In the fall of 1996, asynchronous digital subscriber line technology, capable of $1.544 \mathrm{Mbits} / \mathrm{sec}$ transfer rates, will be available to individual users. This product uses existing telephone copper wires and is reported to be available for $\$ 35$ per month by 1998 (Thyfalut, 1996).

\section{Storage Performance}

The use of high-performance computing and communication equipment leads to the generation of enormous amounts of data. This is painfully apparent for users of dataintensive applications who are held captive by data transfers from disk or by insufficient file space. For cost reasons, systems are configured by making tradeoffs between expensive, high-speed devices of moderate capacity and less expensive, larger capacity, moderate-speed devices.

Typically, these tradeoffs are made between disk and tape storage devices. There are several vendors who offer tape autoloader or library systems that combine disk and tape technologies that are integrated and transparent to the user. In this scheme, the entire file system is actually stored on tape and the disks act as a higher performance cache for user files. On a periodic basis (e.g., once a day), software automatically migrates the files from disk to a robot-controlled tape system. Then the software decides to remove certain files from the disk on the basis of factors such as the file's size, its last modification date, and how full the file system is. The typical performance of 
these devices is on the order of $5 \mathrm{MB} / \mathrm{sec}$ for tape-to-disk transfers and $100 \mathrm{MB} / \mathrm{sec}$ for disk-to-memory transfers.

In terms of disk products, the overall industry trend is toward developing devices of larger capacity rather than higher speed. In terms of tape products, both increased capacity and speed are emphasized in announced products. Digital linear tape (DLT) drives will soon have a capacity of $35 \mathrm{~GB}$ and, when configured in tape arrays, are reported to have fourfold increases over current performance (Simpson, 1996).

\section{Memory Performance}

Once the desired data have reached the computer's memory, system performance is influenced by how the memory is topologically connected to the processors and how fast data are transferred on those connections. Herein lies a major difference between commercially available high-performance computer architectures. Figure 1 displays three common memory-processor organization schemes: (a) bus-based architecture, (b) symmetric multiprocessors, and (c) network-based multiprocessors.

In bus-based architectures, the processor shares a common path to memory (i.e., bus). Cost is a major advantage of this architecture. The number of electrical connections is reduced, and the computer is therefore easier and cheaper to manufacture. Unfortunately, the processordemanded volume of data traffic on the bus is typically more than it is capable of handling. This results in contention along the bus and, ultimately, in reduced performance. This memory-processor configuration works reasonably well for a small number of processors, but if more than four processors are connected to a single bus, other engineering measures must take place to maintain performance.

Symmetric multiprocessors (Figure $1 \mathrm{~b}$ ) attempt to overcome problems in data-traffic congestion by providing many more data paths to memory. In this configuration,

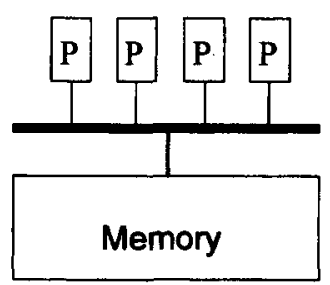

(a)

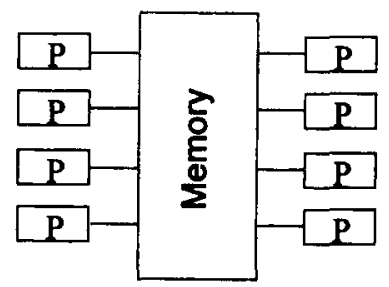

(b)

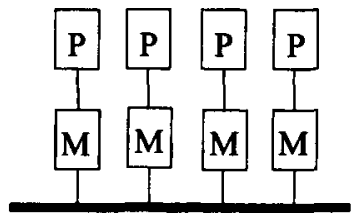

(c)

Figure 1. Three common memory-processor connectivity architectures. $M$ and $P$ signify memory and processors, respectively: (a) bus-based architecture, (b) symmetric multiprocessors, and (c) network-based multiprocessors. each processor can access any memory address in the same amount of time as any other processor. Therefore, this architecture is referred to as a flat-memory, symmetric multiprocessor. The processors access memory through a series of switches that lead to a memory bank. To sustain performance, the required number of memory banks is typically $2^{n+1}$, where $n$ is the number of processors sharing the memory. Cost and packaging-technology factors eventually limit the number of processors to 64 in this configuration. Some high-performance workstations and most vector computers are symmetric multiprocessors.

In a network-based architecture, each processor is directly connected to its own local memory, which communicates with other processors through a combination of hardware- and software-mediated connections. The manner in which these processing elements are connected to each other depends upon the manufacturer. Clustered workstations are typically configured on a ring, while massively parallel processing (MPP) machines have been manufactured using hypercube, two-dimensional rectangular mesh and three-dimensional torus topologies. The allure of these configurations lies in their potential to deliver performance through parallel processing at a price much lower than that of the equivalent performance of a symmetric multiprocessor. Unfortunately, after a decade of intense effort, the potential of these machines has been realized only in a limited number of scientific disciplines. In the next section, some reasons for the apparent lack of success, as well as Cray Research, Inc.'s (CRI's) approach to these hurdles, are presented.

\section{Parallel Processing Challenges}

By considering what it takes to achieve parallelprocessing performance, it is not difficult to see why progress has been limited. For example, assume that an application can perfectly utilize all processors on the machine for $90 \%$ of the time and that the application runs on one processor for $1,000 \mathrm{~h}$. How long will the application take to finish on a 256-processor MPP? The portion of the application that can use all 256 processors will require $3.52 \mathrm{~h}$ (i.e., $900 \mathrm{~h} / 256$ ), but the remaining serial portion will require $100 \mathrm{~h}$ to complete, for a total of $103.52 \mathrm{~h}$. Thus, the application runs on 256 processors only 9.66 times faster than it does on a single processor. Likewise, for applications with $95 \%$ and $99 \%$ parallelism, the speedups would be 18.6 and 72.1 on 256 processors, respectively. While a 72 times speedup is an achievement, the application still utilizes only $28 \%$ of the machine. It is clear that for applications to perform on MPP platforms, they must be able to execute in a parallel fashion for a large percentage of their execution time.

One promising approach to this technical problem (being pursued at CRI) is to design application solutions that can effectively use smaller numbers of processors, but multiple copies of the application (with different data sets) are run at the same time. In this scheme, parallelism on the job-submission level is exploited, which allows for more flexible hardware designs that incorporate share memory on a smaller number of processors. 
The preceding discussion assumes that the parallel performance is perfect, which in reality will be less than perfect for both hardware and software reasons. Some of the most common reasons for performance degradation are slow interprocessor communication, inefficient program synchronization, and low individual processor performance. CRI's approach to the interprocessor-communication issues was to engineer hardware that communicated an order of magnitude faster than other network-based platforms. Inefficient program synchronization can result from poor hardware performance or from the application. Again CRI's approach was to provide extremely fast synchronization hardware as well as performance analysis tools that allowed programmers to detect inefficiencies within the application. Poor individual processor performance results from a host of issues related to the complexities of commodity microprocessors employed in most network-based architectures. To help mitigate some of these problems, CRI has added hardware features that improve the flow of data from local memory to the microprocessor.

Despite these technical barriers, progress in parallel processing is being made. As researchers from a variety of disciplines wrestle with the complexities of their specific systems, they continually advance the field of highperformance computing and communication.

\section{REFERENCES}

MAYER, J. H. (1996, April). Client with fat bandwidth future takes fibre Reseller Management, pp. 62-66.

SiMPSON, D. (1996, April 15). It's time to reevaluate your tape options. Data Mation, pp. 58-62.

TADJER, R. (1996, March 25). ISDN devices: Taming digital lines. Communications Week, pp. 63-66.

Thyfalut, M. E. (1996, April 8). High bandwidth at low price. Information Week, p. 16. 